Proyecciones Journal of Mathematics

Vol. 40, No 5, pp. 1267-1277, October 2021.

Universidad Católica del Norte

Antofagasta - Chile

\title{
On the total irregularity strength of convex polytope graphs
}

\author{
Syed Ahtsham Ul Haq Bokhary \\ Bahauddin Zakaria University, Pakistan \\ Muhammad Imran \\ United Arab Emirates University, United Arab Emirates \\ and \\ Usman Ali \\ Bahauddin Zakaria University, Pakistan \\ Received : January 2020. Accepted: April 2021
}

\begin{abstract}
$A$ vertex (edge) irregular total $k$-labeling $\phi$ of a graph $G$ is a labeling of the vertices and edges of $G$ with labels from the set $\{1,2, \ldots, k\}$ in such a way that any two different vertices (edges) have distinct weights. Here, the weight of a vertex $x$ in $G$ is the sum of the label of $x$ and the labels of all edges incident with the vertex $x$, whereas the weight of an edge is the sum of label of the edge and the vertices incident to that edge. The minimum $k$ for which the graph $G$ has a vertex (edge) irregular total $k$-labeling is called the total vertex (edge) irregularity strength of $G$.

In this paper, we are dealing with infinite classes of convex polytopes generated by prism graph and antiprism graph. We have determined the exact value of their total vertex irregularity strength and total edge irregularity strength.
\end{abstract}

2010 Mathematics Subject Classification: $05 C 78$.

Keywords: Irregular assignment, vertex irregular total $k$-labeling, irregularity strength, convex polytopes. 


\section{Introduction}

The graph labeling has caught the attention of many authors and many new labeling results appear every year. This popularity is not only due to the mathematical challenges of graph labeling, but also for the wide range of its application, for instance X-ray, crystallography, coding theory, radar, astronomy, circuit design, network design and communication design. Bloom and Golomb studied applications of graph labelings to other branches of science $[5,6]$.

Let us consider a simple (without loops and multiple edges) undirected graph $G=(V, E)$. A total labeling is defined as a labeling in which all the vertices and edges are labeled. For a graph $G$, we define a labeling $\phi: V \cup E \rightarrow\{1,2, \ldots, k\}$ to be a vertex irregular total $k$-labeling of the graph $G$ if for every two different vertices $x$ and $y$ of $G, w t(x) \neq w t(y)$ where the weight of a vertex $x$ in the labeling $\phi$ is

$$
w t(x)=\phi(x)+\sum_{y \in N(x)} \phi(x y),
$$

where $N(x)$ is the set of neighbors of $x$. On the other hand, $\phi$ is said to be edge irregular total labeling if for any two distinct edges $e$ and $f$ of $G$, $w t(e) \neq w t(f)$, where the weight of an edge $e$ in the labeling $\phi$ is

$$
w t(e)=\phi(e)+\phi(x)+\phi(y)
$$

where $x$ and $y$ are vertices adjacent to edge $e$. In [2] Bača et al. defined a new graph invariant, called the total vertex (edge) irregularity strength of $G, \operatorname{tvs}(G)(\operatorname{tes}(G))$, that is the minimum $k$ for which the graph $G$ has a vertex (edge) irregular total $k$-labeling.

The original motivation for the definition of the total vertex (edge) irregularity strength came from irregular assignments and the irregularity strength of graphs introduced in [9] by Chartrand et al. and studied by numerous in $[7,10,11,12,14]$.

An irregular assignment is a $k$-labeling of the edges

$$
f: E \rightarrow\{1,2, \ldots, k\}
$$

such that the vertex weights

$$
w(x)=\sum_{y \in N(x)} f(x y)
$$


are different for all vertices of $G$, and the smallest $k$ for which there is an irregular assignment is the irregularity strength, $s(G)$.

The irregularity strength $s(G)$ can be interpreted as the smallest integer $k$ for which $G$ can be turned into a multigraph $G^{\prime}$ by replacing each edge by a set of at most $k$ parallel edges, such that the degrees of the vertices in $G^{\prime}$ are all different.

It is easy to see that the irregularity strength $s(G)$ of a graph $G$ is defined only for graphs containing at most one isolated vertex and no connected component of order 2. On the other hand, the total vertex irregularity strength $\operatorname{tvs}(G)$ is defined for every graph $G$. Thus, the total labeling $\phi$ is a vertex irregular total labeling and for graphs with no component of order $\leq 2, \operatorname{tvs}(G) \leq s(G)$. Nierhoff [16] proved that for every $(p, q)$-graph $G$ (i.e. the graph with $p$ vertices and $q$ edges) with no component of order at most 2 and $G \neq K_{3}$, the irregularity strength $s(G) \leq p-1$. From this result it follows that

$$
\operatorname{tvs}(G) \leq p-1 .
$$

In [2], Bača et al. put forward the lower bounds for total vertex irregularity strength and total edge irregularity strength in terms of maximum degree $\Delta$, minimum degree $\delta,|V(G)|=p$ and $|E(G)|=q$, which may be stated as the theorems 1 and 2 .

Theorem 1.1. Let $G$ be a $(p, q)$-graph having minimum degree $\delta=\delta(G)$ and maximum degree $\Delta=\Delta(G)$. Then the lower bound for $\operatorname{tvs}(G)$ is

$$
\operatorname{tvs}(G) \geq\left\lceil\frac{p+\delta}{\Delta+1}\right\rceil,
$$

Theorem 1.2. The lower bound for tes $(G)$ is

$$
\operatorname{tes}(G) \geq \max \left\{\left\lceil\frac{q+2}{3}\right\rceil,\left\lceil\frac{\Delta+1}{2}\right\rceil\right\}
$$

For graphs with no component of order $\leq 2$, Bača et al. in [2] proved the following inequality:

$$
\operatorname{tvs}(G) \leq p-1-\left\lceil\frac{p-2}{\Delta+1}\right\rceil .
$$

These results were then improved by Przybylo in [17] for sparse graphs and for graphs with large minimum degree. In [18, 19], Wijaya et al. 
found the exact values of the total vertex irregularity strength of wheels, fans, suns, friendship graphs and complete bipartite graphs. More recently, Bokhary et al. [8] determined an exact value of the total vertex irregularity strength for some families of cubic graphs.

In [2], Baca et al. determined the total vertex irregularity strength of prisms. The prism and antiprism are the Archimedian convex polytopes defined e.g. in [15]. Ahmad determined the total vertex irregularity strength of some classes of convex polytopes in [1]. In this paper, we determined an exact value of total vertex irregularity strength as well as total edge irregularity strength for some infinite classes of convex polytopes.

\section{The graph of convex polytope $S_{n}$}

For $n \geq 3$, the graph of convex polytope $S_{n}$ (Fig.1) consisting of $2 n 3$ sided faces, $2 n 4$-sided faces and a pair of $n$-sided faces, is obtained by the combination of the graph of convex polytope $R_{n}$ [4] and the graph of a prism $D_{n}$. We have

$$
V\left(S_{n}\right)=\left\{a_{i}, b_{i}, c_{i}, d_{i} \mid 1 \leq i \leq n\right\}
$$

and

$$
\mathrm{E}\left(\mathrm{S}_{n}\right)=\left\{a_{i} a_{i+1}, b_{i} b_{i+1}, c_{i} c_{i+1}, d_{i} d_{i+1} \mid 1 \leq i \leq n\right\}
$$

$\cup\left\{a_{i+1} b_{i}, a_{i} b_{i}, b_{i} c_{i}, c_{i} d_{i} \mid 1 \leq i \leq n\right\}$.

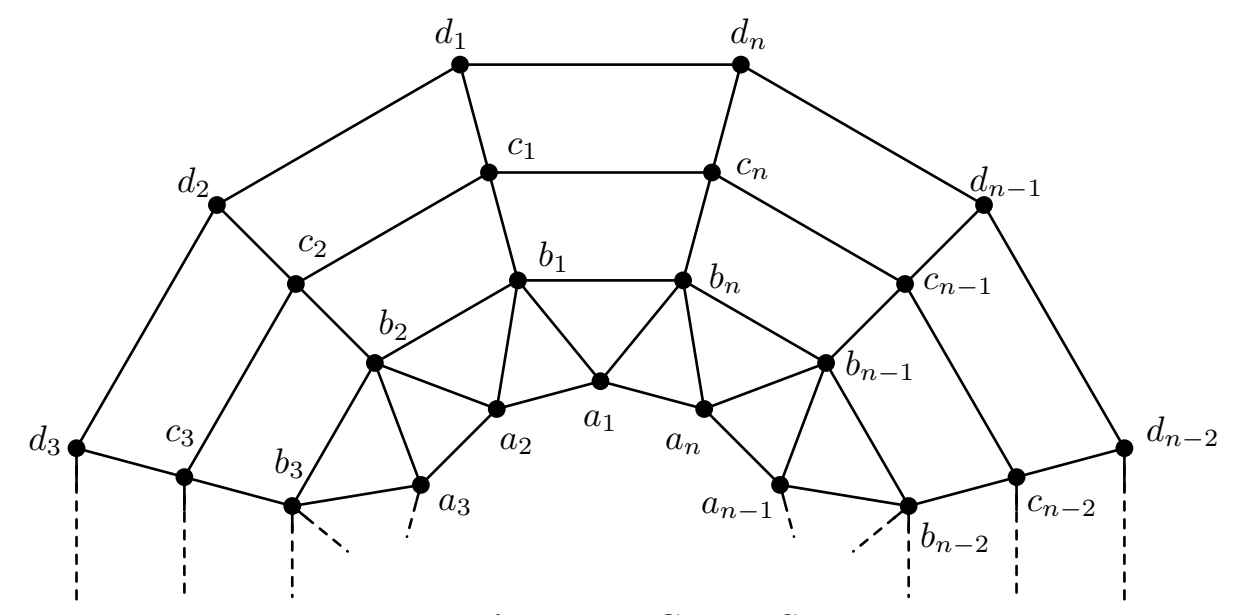

Figure 1: Graph $S_{n}$

The graph of convex polytope $S_{n}$ can also be obtained from the graph of convex polytope $Q_{n}$ defined in [4] by adding the edges $a_{i+1} b_{i}, c_{i} c_{i+1}$ 
and then deleting the edges $b_{i+1} c_{i}$. i.e., $V\left(S_{n}\right)=V\left(Q_{n}\right)$ and $E\left(S_{n}\right)=$ $\left(E\left(Q_{n}\right) \cup\left\{a_{i+1} b_{i}, c_{i} c_{i+1} \mid 1 \leq i \leq n\right\}\right) \backslash\left\{b_{i+1} c_{i} \mid 1 \leq i \leq n\right\}$.

The total vertex irregularity strength of graph of convex polytope $R_{n}$ and graph of a prism $D_{n}$ have been studied in [1] and [2], respectively. In the next two theorems, we find the total vertex irregularity strength and total edge irregularity strength of the graph of convex polytope $S_{n}$.

Theorem 2.1. For $n \geq 3$, the total vertex irregularity strength of $S_{n}$ is $\left\lceil\frac{4 n+3}{6}\right\rceil$.

Proof. From inequality (1.2) it follows that $\operatorname{tvs}\left(S_{n}\right) \geq\left\lceil\frac{4 n+3}{6}\right]$.

Let $k=\left\lceil\frac{4 n+3}{6}\right\rceil$. It is sufficient to define an irregular total $k$-labeling as following. For $1 \leq i \leq n$, let

$$
\begin{aligned}
\phi\left(d_{i}\right)=\phi\left(a_{i}\right) & =\left\lceil\frac{i}{2}\right\rceil \\
\phi\left(d_{i} d_{i+1}\right)=\phi\left(c_{i}\right) & =1 \\
\phi\left(d_{i} c_{i}\right) & =\left\lceil\frac{i+1}{2}\right\rceil \\
\phi\left(c_{i} c_{i+1}\right) & =\left\lceil\frac{n+2}{3}\right\rceil \\
\phi\left(c_{i} b_{i}\right) & =\left\lceil\frac{i}{2}\right\rceil+\left\lceil\frac{n-3}{3}\right\rceil \\
\phi\left(b_{i}\right)=\phi\left(b_{i} a_{i+1}\right)=\phi\left(b_{i} b_{i+1}\right) & =k \\
\phi\left(b_{i} a_{i}\right) & =\left\lceil\frac{i+1}{2}\right\rceil+1 \\
\phi\left(a_{i} a_{i+1}\right) & =\left\lceil\frac{4 n+1}{6}\right\rceil .
\end{aligned}
$$

One can easily check that different vertices in $S_{n}$ have different weights and so $\operatorname{tvs}\left(S_{n}\right) \leq k$. Hence, Inequality (1.2) implies that $\operatorname{tvs}\left(S_{n}\right)=k$.

Theorem 2.2. For $n \geq 3$, the total edge irregularity strength of $S_{n}$ is $\left\lceil\frac{8 n+2}{3}\right\rceil$.

Proof. From inequality (1.3) it follows that $\operatorname{tes}\left(S_{n}\right) \geq\left\lceil\frac{8 n+2}{3}\right\rceil$.

Let $\left\lceil\frac{8 n+2}{3}\right\rceil=k$ and define an irregular total $k$-labeling in order to show that $k$ is an upper bound for tes $\left(S_{n}\right)$. 
For $1 \leq i \leq n$,

$\phi\left(d_{i}\right)=1$,

$\phi\left(d_{i} d_{i+1}\right)=\phi\left(d_{i} c_{i}\right)=\phi\left(c_{i} c_{i+1}\right)=\phi\left(c_{i} b_{i}\right)=\phi\left(b_{i} b_{i+1}\right)=i$,

$\phi\left(c_{i}\right)=n+1$,

$\phi\left(b_{i}\right)=2 n+1$,

$\phi\left(a_{i}\right)=k$,

$\phi\left(b_{i} a_{i}\right)=2 i-1+\left\lceil\frac{n-1}{3}\right\rceil$,

$\phi\left(b_{i} a_{i+1}\right)=2 i+\left\lceil\frac{n-1}{3}\right\rceil$,

$\phi\left(a_{i} a_{i+1}\right)=n+i+2\left\lceil\frac{n-1}{3}\right\rceil$.

One can easily check that distinct edges in $S_{n}$ have different weights and so tes $\left(S_{n}\right) \leq k$. Hence, Inequality (1.3) implies that $\operatorname{tes}\left(S_{n}\right)=k$.

\section{The graph of convex polytope $T_{n}$}

For $n \geq 3$, the graph of convex polytope $T_{n}$ (Fig. 2) consists of $4 n 3$-sided faces, $n 4$-sided faces and a pair of $n$-sided faces, and is obtained by the combination of the graph of convex polytope $R_{n}$ [4] and the graph of an antiprism $A_{n}[3]$. We have

$$
V\left(T_{n}\right)=\left\{a_{i}, b_{i}, c_{i}, d_{i} \mid 1 \leq i \leq n\right\}
$$

and

$$
\mathrm{E}\left(\mathrm{T}_{n}\right)=\left\{a_{i} a_{i+1}, b_{i} b_{i+1}, c_{i} c_{i+1}, d_{i} d_{i+1} \mid 1 \leq i \leq n\right\}
$$

$\cup\left\{a_{i+1} b_{i}, a_{i} b_{i}, b_{i} c_{i}, c_{i} d_{i}, c_{i+1} d_{i} \mid 1 \leq i \leq n\right\}$.

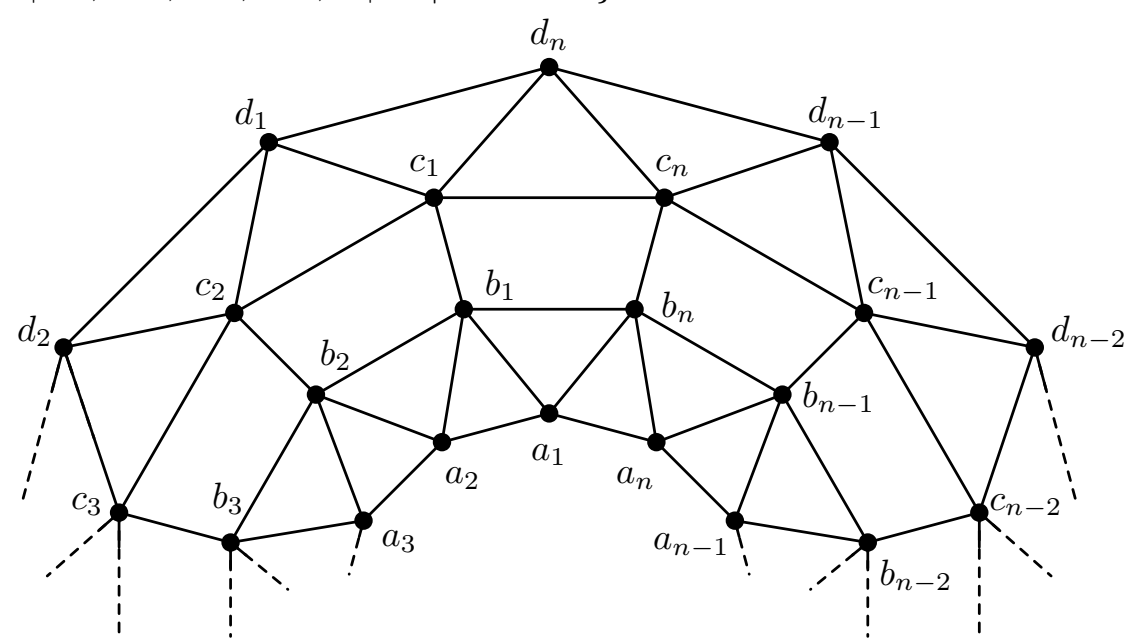

Figure 2: Graph $T_{n}$ 
The graph of convex polytope $T_{n}$ can also be obtained from the graph of convex polytope $S_{n}$ by adding the edges $c_{i+1} d_{i}$. i.e., $V\left(T_{n}\right)=V\left(S_{n}\right)$ and $E\left(T_{n}\right)=\left(E\left(S_{n}\right) \cup\left\{a_{i+1} b_{i}, c_{i} c_{i+1}, c_{i+1} d_{i} \mid 1 \leq i \leq n\right\}\right) \backslash\left\{b_{i+1} c_{i} \mid 1 \leq i \leq n\right\}$.

The total vertex irregularity strength of the graph of convex polytope $R_{n}$ and graph of an antiprism $A_{n}$ have been studied in [1]. In the next two theorems we find the total vertex irregularity strength and total edge irregularity strength of the graph of convex polytope $T_{s} n$.

Theorem 3.1. For $n \geq 6$, the total vertex irregularity strength of $T_{n}$ is $\left\lceil\frac{4 n+4}{6}\right\rceil$.

Proof. Let $k=\left\lceil\frac{4 n+4}{6}\right\rceil$. From inequality (1.2) it is clear that $t v s\left(T_{n}\right) \geq$ $k$. Now we will define an irregular total $k$-labeling in order to show that $k$ is an upper bound for $\operatorname{tvs}\left(T_{n}\right)$.

For $1 \leq i \leq n$,

$$
\phi\left(d_{i}\right)=\left\lceil\frac{i}{2}\right\rceil,
$$

$\phi\left(d_{i} d_{i+1}\right)=\phi\left(d_{i} c_{i+1}\right)=\phi\left(a_{i} a_{i+1}\right)=1$,

$\phi\left(d_{i} c_{i}\right)=\left\lceil\frac{i+1}{2}\right\rceil$,

$\phi\left(c_{i}\right)=\left\lceil\frac{n+1}{2}\right\rceil$,

$\phi\left(c_{i} b_{i}\right)=\left\lceil\frac{i}{2}\right\rceil+\left\lfloor\frac{n+1}{6}\right\rfloor$,

$\phi\left(c_{i} c_{i+1}\right)=\phi\left(b_{i}\right)=\phi\left(b_{i} a_{i+1}\right)=\phi\left(b_{i} b_{i+1}\right)=k$,

$\phi\left(b_{i} a_{i}\right)=\left\lceil\frac{i+1}{2}\right\rceil+\left\lfloor\frac{n+4}{6}\right\rfloor$,

$\phi\left(a_{i}\right)=\left\lceil\frac{i}{2}\right\rceil+\left\lfloor\frac{n+1}{6}\right\rfloor$.

One can easily check that distinct vertices in $T_{n}$ have different weights and so $\operatorname{tvs}\left(T_{n}\right) \leq k$. Hence, Inequality (1.2) implies that $\operatorname{tvs}\left(T_{n}\right)=k$.

Theorem 3.2. For $n \geq 3$, the total edge irregularity strength of $T_{n}$ is $\left\lceil\frac{9 n+2}{3}\right\rceil$.

Proof. Let $k=\left\lceil\frac{9 n+2}{3}\right\rceil$. From inequality (1.3), we have $\operatorname{tvs}\left(T_{n}\right) \geq k$. To prove the equality, we define an irregular total $k$-labeling as follows:

For $1 \leq i \leq n$, $\phi\left(d_{i}\right)=1$,

$\phi\left(d_{i} d_{i+1}\right)=i+1$,

$\phi\left(c_{i}\right)=n+1$,

$\phi\left(d_{i} c_{i}\right)=2 i-1$,

$\phi\left(d_{i} c_{i+1}\right)=2 i$, 
$\phi\left(c_{i} c_{i+1}\right)=\phi\left(c_{i} b_{i}\right)=\phi\left(b_{i} b_{i+1}\right)=\phi\left(a_{i} a_{i+1}\right)=n+i$,

$\phi\left(b_{i}\right)=2 n+1$,

$\phi\left(a_{i}\right)=k$,

$\phi\left(b_{i} a_{i}\right)=2 n+2 i-1$,

$\phi\left(b_{i} a_{i+1}\right)=2 n+2 i$.

One can easily check that distinct edges in $T_{n}$ have different weights and so tes $\left(T_{n}\right) \leq k$. Hence, Inequality (1.3) implies that $\operatorname{tes}\left(T_{n}\right)=k$.

\section{The graph of convex polytope $U_{n}$}

For $n \geq 3$, the graph of convex polytope $U_{n}$ (Fig. 3) consists of $n 4$-sided faces, $2 n$-sided faces and a pair of $n$-sided faces, and is obtained as a combination of the graph of convex polytope $D_{n}[3]$ and graph of a prism $D_{n}$. We have

$$
V\left(U_{n}\right)=\left\{a_{i}, b_{i}, c_{i}, d_{i}, e_{i} \mid 1 \leq i \leq n\right\}
$$

and

$$
\mathrm{E}\left(\mathrm{U}_{n}\right)=\left\{a_{i} a_{i+1}, b_{i} b_{i+1}, e_{i} e_{i+1} \mid 1 \leq i \leq n\right\}
$$

$\cup\left\{a_{i} b_{i}, b_{i} c_{i}, c_{i} d_{i}, d_{i} e_{i}, c_{i+1} d_{i} \mid 1 \leq i \leq n\right\}$.

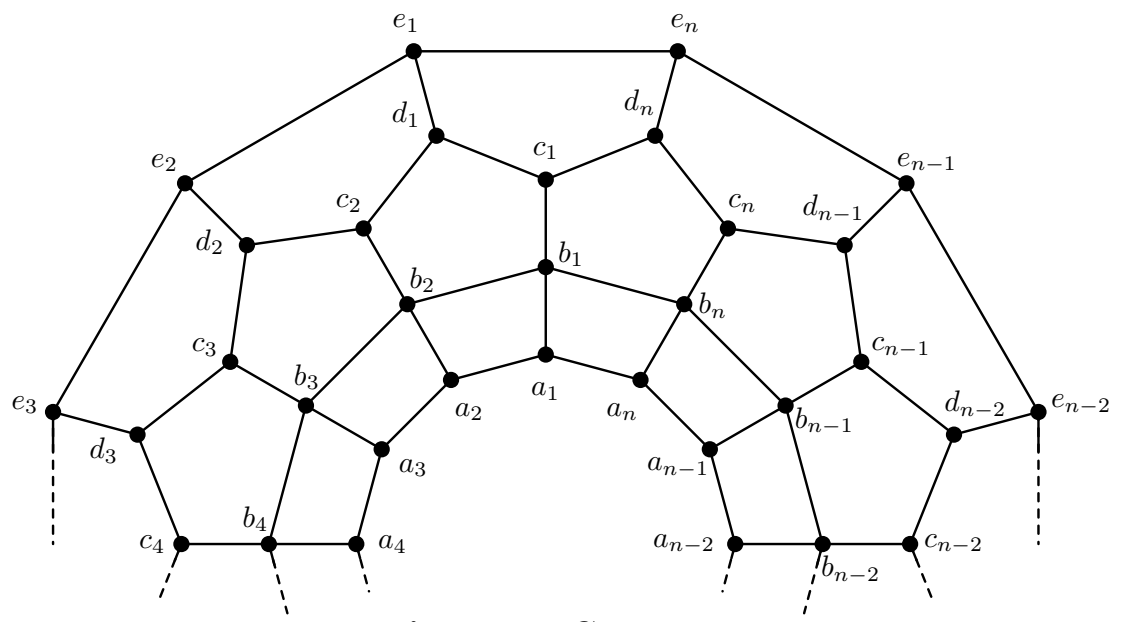

Figure 3: Graph $U_{n}$

The graph of convex polytopes $D_{n}$ and graph of a prism $D_{n}$ have been studied in [1] and [2]. In the next two theorems, we find the The total vertex and edge irregularity strength of the graph of convex polytope $U_{n}$.

Theorem 4.1. For $n \geq 5$, the total vertex irregularity strength of $U_{n}$ is $\left\lceil\frac{5 n+3}{5}\right\rceil$. 
Proof. Let $k=\left\lceil\frac{5 n+3}{5}\right\rceil$. To show that $\operatorname{tvs}\left(U_{n}\right)=k$, we define the following $k$-labelling:

For $1 \leq i \leq n$,

$\phi\left(e_{i}\right)=\phi\left(e_{i} e_{i+1}\right)=\phi\left(d_{i}\right)=1$,

$\phi\left(e_{i} d_{i}\right)=\phi\left(b_{i}\right)=\phi\left(c_{i}\right)=i$

$\phi\left(d_{i} c_{i+1}\right)=\phi\left(d_{i} c_{i}\right)=\left\lceil\frac{n+2}{2}\right\rceil$,

$\phi\left(c_{i} b_{i}\right)=\phi\left(b_{i} a_{i}\right)=\phi\left(b_{i} b_{i+1}\right)=\phi\left(a_{i} a_{i+1}\right)=k$,

$\phi\left(a_{i}\right)=i+1$.

From the above labeling, it is clear that every vertex has distinct weight. Thus, $\phi$ is a vertex irregular $k$-labelling and therefore $\operatorname{tvs}\left(U_{n}\right) \leq k$. Hence, Inequality (1.2) implies that $\operatorname{tvs}\left(U_{n}\right)=k$.

Theorem 4.2. For $n \geq 3$, the total edge irregularity strength of $U_{n}$ is $\left\lceil\frac{8 n+2}{3}\right\rceil$.

Proof. Let $k=\left\lceil\frac{8 n+2}{3}\right\rceil$. Define an irregular total $k$-labeling as follows.

For $1 \leq i \leq n$,

$\phi\left(e_{i}\right)=1$,

$\phi\left(e_{i} e_{i+1}\right)=\phi\left(e_{i} d_{i}\right)=i$,

$\phi\left(d_{i}\right)=\phi\left(c_{i}\right)=n+1$,

$\phi\left(d_{i} c_{i}\right)=2 i-1$,

$\phi\left(d_{i} c_{i+1}\right)=2 i$

$\phi\left(b_{i}\right)=\left\lceil\frac{7 n+2}{3}\right\rceil$,

$\phi\left(c_{i} b_{i}\right)=\left\lceil\frac{2 n-1}{3}\right\rceil+i$,

$\phi\left(b_{i} b_{i+1}\right)= \begin{cases}\left\lceil\frac{n-2}{3}\right\rceil+i & \text { for } n \equiv 2(\bmod 3) \\ \left\lceil\frac{n}{3}\right\rceil+i & \text { for } n \equiv 0,1(\bmod 3)\end{cases}$

$\phi\left(a_{i}\right)=k$,

$\phi\left(b_{i} a_{i}\right)=n+i$,

$\phi\left(a_{i} a_{i+1}\right)=2\left\lceil\frac{n-1}{3}\right\rceil+n+i$.

From the above labeling, it is clear that every edge has distinct weight, so tes $\left(U_{n}\right) \leq k$. Hence, Inequality (1.3) implies that tes $\left(U_{n}\right)=k$.

\section{Acknowledgment}

This research is supported by the Start Up Research Grant 2016 of United Arab Emirates University (Grant No. G00002233)

\section{References}

[1] A. Ahmad, "On vertex irregular total labelings of convex polytope graphs", Utilitas Mathematica, vol. 89, pp. 69-78, 2012. 
[2] M. Bača, S. Jendrol, M. Miller. and J. Ryan, "On irregular total labellings", Discrete Mathematics, vol. 307, pp. 1378-1388, 2007.

[3] M. Bača, "Labellings of two classes of convex polytopes", Utilitas Mathematica, vol. 34, pp. 24-31, 1988.

[4] M. Bača, "On magic labellings of convex polytopes", Annals of Discrete Mathematics, vol. 51, pp. 13-16, 1992.

[5] G. S. Bloom and S. W. Golomb, "Applications of numbered undirected graphs", Proceedings of the IEEE, vol. 65, no. 4, pp. 562-570, 1977.

[6] G. S. Bloom and S. W. Golomb, "Numbered complete graphs, unusual rules, and assorted applications", in Theory and Applications of Graphs, Y. Alavi and D. R. Lick, Eds. Berlin: Springer, 1978, pp. 53-65.

[7] T. Bohman and D. Kravitz, "On the irregularity strength of trees", Journal of Graph Theory, vol. 45, no. 4, pp. 241-254, 2004.

[8] S. A. Bokhary, M. Imran, and A. Ahmad, "On vertex irregular total labeling of some cubic graphs", Utilitas Mathematica, vol. 91, pp. 239-249, 2013.

[9] G. Chartrand, M. S. Jacobson, J. Lehel, O. R. Oellermann, S. Ruiz, and F. Saba, "Irregular networks", Congressus Numerantium, vol. 64, pp. 187-192, 1988.

[10] R. J. Faudree, M. S. Jacobson, J. Lehel. and R. H. Schlep, "Irregular networks, regular graphs and integer matrices with distinct row and column sums", Discrete Mathematics, vol. 76, no. 3, pp. 223-240, 1988.

[11] A. Frieze, R. J. Gould, M. Karonski, and F. Pfender, "On graph irregularity strength", Journal of Graph Theory, vol. 41, no. 2, pp. 120-137, 2002.

[12] A. Gyárfás, "The irregularity strength of $K_{m, m}$ is 4 for odd m", Discrete Mathematics, vol. 71, pp. 273-274, 1988.

[13] M. Imran, S. A. Bokhary, and A. Q. Baig, "On families of convex polytopes with constant metric dimension", Computers \& Mathematics with Applications, vol. 60, pp. 2629-2638, 2010.

[14] S. Jendrol, M. Tkáč and Z. Tuza, "The irregularity strength and cost of the union of cliques", Discrete Mathematics, vol. 150, pp. 179-186, 1996. 
[15] E.Jucovič, Konvexné mnohosteny. Bratislava: Veda, 1981.

[16] T. Nierhoff, "A tight bound on the irregularity strength of graphs", SIAM Journal on Discrete Mathematics, vol. 13, no. 3, pp. 313-323, 2000.

[17] J. Przybylo, "Linear bound on the irregularity strength and the total vertex irregularity strength of graphs", SIAM Journal on Discrete Mathematics, vol. 23, no. 1, pp. 511-516, 2009.

[18] K. Wijaya and Slamin, "Total vertex irregular labeling of wheels, fans, suns and friendship graphs", Journal of Combinatorial Mathematics and Combinatorial Computing, vol. 65, pp. 103-112, 2008.

[19] K. Wijaya, Slamin, S. Supangken and S. Jendrol, "Total vertex irregular labeling of complete bipartite graphs", Journal of Combinatorial Mathematics and Combinatorial Computing, vol. 55, pp. 129-136, 2005.

\section{Syed Ahtsham Ul Haq Bokhary}

Centre for Advanced Studies in Pure and Applied Mathematics Bahauddin Zakaria University,

Multan,

Pakistan

e-mail: sihtsham@gmail.com

Corresponding author

\section{Muhammad Imran}

Department of Mathematical Sciences

United Arab Emirates University

P. O. Box 15551, Al Ain

United Arab Emirates

e-mail: imrandhab@gmail.com

and

\section{Usman Ali}

Centre for Advanced Studies in Pure and Applied Mathematics Bahauddin Zakaria University,

Multan,

Pakistan

e-mail: uali@bzu.edu.pk 